\title{
THE $p$-NORM JOINT SPECTRAL RADIUS FOR EVEN INTEGERS
}

\section{Ding-Xuan Zhou}

ABSTRACT. Let $\mathcal{A}$ be a multiset of square matrices of the same size and $\|\cdot\|$ be a matrix norm. For $0<p<\infty$, the $p$-norm joint spectral radius $\rho_{p}(\mathcal{A})$ of $\mathcal{A}$ is defined by

$$
\rho_{p}(\mathcal{A}):=\lim _{n \rightarrow \infty}\left(\sum_{A_{1}, \ldots, A_{n} \in \mathcal{A}}\left\|A_{1} \cdots A_{n}\right\|^{p}\right)^{1 / n p} .
$$

This concept plays an important role in the investigation of wavelets and subdivision schemes.

The purpose of this paper is to give a formula for computing $\rho_{p}(\mathcal{A})$ in terms of the spectral radius of some finite matrix when $p$ is an even integer:

$$
\rho_{2 k}(\mathcal{A})=\left\{\rho\left(\sum_{A \in \mathcal{A}}(\bar{A} \otimes A)^{[k]}\right)\right\}^{1 / 2 k}, \quad k \in \mathbb{N},
$$

where $\bar{A} \otimes A$ is the Kronecker product, and $A^{[1]}=A, A^{[l+1]}=A \otimes A^{[l]}$. This formula is employed to study the uniform joint spectral radius $(p=\infty)$. Examples including multiple refinable Hermite interpolants will be provided to illustrate the general theory.

\section{Introduction}

The spectral radius of a complex matrix $A$ is defined by

$$
\rho(A):=\max \{|\lambda|: \lambda \text { is an eigenvalue of } A\} .
$$

Due to many practical applications, this definition has been extended in various ways to sets (multisets) of matrices. In this paper we consider one extension called the $p$-norm joint spectral radius.

Let $\|\cdot\|$ be a matrix norm on $\mathbb{C}^{M \times M}$, the set of all $M \times M$ complex matrices. It is well known that for any $M \times M$ matrix $A$,

$$
\rho(A)=\lim _{n \rightarrow \infty}\left\|A^{n}\right\|^{1 / n} .
$$

This tells us that the $p$-norm joint spectral radius defined below is a natural extension of the spectral radius.

Let $\mathcal{A}:=\left\{A_{1}, \ldots, A_{d}\right\}$ be a multiset of $M \times M$ complex matrices. Replacing $\left\|A^{n}\right\|$ in (1.1), we set $\left\|\mathcal{A}^{n}\right\|_{p}$ as the $\ell_{p}$ norm (seminorm) of the norm sequence of all products

Received April 30, 1997, revised October 22, 1997.

1991 Mathematics Subject Classification: Primary 42C15, Secondary 39B12, 41A25, 65F15.

Key words and phrases: $p$-norm joint spectral radius, Kronecker product, string vec-function, multiple refinable functions, wavelets, multiple refinable Hermite interpolant. 
(in all orders) of $n$ matrices from $\mathcal{A}$ :

$$
\left\|\mathcal{A}^{n}\right\|_{p}:= \begin{cases}\max \left\{\left\|A_{\epsilon_{1}} \cdots A_{\epsilon_{n}}\right\|: \epsilon_{1}, \ldots, \epsilon_{n} \in\{1, \ldots, d\}\right\}, & \text { if } p=\infty \\ \left\{\sum_{\epsilon_{1}, \ldots, \epsilon_{n} \in\{1, \ldots, d\}}\left\|A_{\epsilon_{1}} \cdots A_{\epsilon_{n}}\right\|^{p}\right\}^{1 / p}, & \text { if } 0<p<\infty\end{cases}
$$

Then the $p$-norm joint spectral radius $\rho_{p}(\mathcal{A})$ is defined to be

$$
\rho_{p}(\mathcal{A}):=\lim _{n \rightarrow \infty}\left\|\mathcal{A}^{n}\right\|_{p}^{1 / n} .
$$

It is a classical fact that this limit exists and equals the infimum:

$$
\lim _{n \rightarrow \infty}\left\|\mathcal{A}^{n}\right\|_{p}^{1 / n}=\inf _{n \in \mathbb{N}}\left\|\mathcal{A}^{n}\right\|_{p}^{1 / n}
$$

Clearly, $\rho_{p}(\mathcal{A})$ is independent of the choice of the matrix norm $\|\cdot\|$.

If $\mathcal{A}$ consists of a single matrix $A$, then $\rho_{p}(\mathcal{A})=\rho(A)$. Moreover, if $A_{1}=\cdots=$ $A_{d}=A$, then $\rho_{p}(\mathcal{A})=d^{1 / p} \rho(A)$ for $0<p \leq \infty$. It is obvious that each $\rho\left(A_{j}\right) \leq \rho_{\infty}(\mathcal{A})$ since $\rho_{\infty}(\mathcal{A})$ includes the power $\left(A_{j}\right)^{n}$, where we choose the same matrix $A_{j}$ each time by $\epsilon_{i}=j$.

The concept of uniform joint spectral radius $(p=\infty)$ was introduced by Rota and Strang [22] in 1960. It was Daubechies and Lagarias [3] who rediscovered this concept and showed the fundamental connection to refinement equations. Their paper [3] initiated the application of joint spectral radius to the research of regularity and existence for refinement equations. The mean spectral radius $(p=1)$ was defined by Wang [25], who studied $L_{1}$ solutions of refinement equations. The concept of $p$-norm joint spectral radius with $1 \leq p \leq \infty$ was introduced by Jia in [12] and was used implicitly by Lau and Wang [20] independently. This concept plays an important role in the investigation of wavelets. See the unified approaches of Jia [12] to the smoothness analysis in [24] and the convergence of subdivision schemes (see [5] for $p=\infty)$. For recent applications in finding critical smoothness of multiple wavelets and multivariate wavelets, we refer the reader to Han and Jia [9, 13], Jia, Riemenschneider, and Zhou [16, 17], and Micchelli and Sauer [21].

What is disappointing about the joint spectral radius is that the limit in (1.2) is reached very slowly. Except for some special cases such as simultaneously triangularizable matrices (see, e.g., $[10,16])$, we can hardly compute $\rho_{p}(\mathcal{A})$ using (1.2). According to the analysis in [23], the uniform joint spectral radius $(p=\infty)$ cannot be approximated in polynomial time. Thus, it is desirable to find fast ways to compute the $p$-norm joint spectral radius.

In wavelet analysis, the matrices involving the $p$-norm joint spectral radius have special structures. As an example, consider a sequence $a$ supported in $[0, N]$ with $N \in \mathbb{N}$. Define two matrices $A_{0}$ and $A_{1}$ in terms of $a$ by

$$
\begin{aligned}
& A_{0}=(a(2 j-l))_{0 \leq j, l \leq N-1}=\left[\begin{array}{cccc}
a(0) & 0 & \cdots & 0 \\
\vdots & & \Lambda
\end{array}\right] \\
& A_{1}=(a(2 j-l+1))_{0 \leq j, l \leq N-1}=\left[\begin{array}{cccc} 
& \Lambda & & \vdots \\
0 & \cdots & 0 & a(N)
\end{array}\right],
\end{aligned}
$$


where $\Lambda$ is an $(N-1) \times(N-1)$ matrix given by

$$
\Lambda=(a(2 j-l))_{1 \leq j, l \leq N-1}=\left[\begin{array}{cccccc}
a(1) & a(0) & 0 & \cdots & & 0 \\
a(3) & a(2) & a(1) & \cdots & & 0 \\
\vdots & \vdots & \vdots & & & \vdots \\
0 & 0 & 0 & \cdots & a(N) & a(N-1)
\end{array}\right] .
$$

Then the two matrices $A_{0}$ and $A_{1}$ share some special properties. In particular, they have $N-1$ common eigenvalues. The $p$-norm joint spectral radius $\rho_{p}\left(A_{0}, A_{1}\right)$ is essential to the analysis of regularity of wavelets and convergence of subdivision schemes. It is well known in wavelet analysis that, because of the special structures, the 2-norm joint spectral radius of $\left\{A_{0}, A_{1}\right\}$ equals the square root of the spectral radius of a finite matrix:

$$
\rho_{2}\left(A_{0}, A_{1}\right)=\sqrt{\rho(F)}
$$

where $F$ is the $(2 N+1) \times(2 N+1)$ matrix given by

$$
F=\left(\sum_{m=0}^{N} a(m+j-2 l) \overline{a(m)}\right)_{-N \leq j, l \leq N} .
$$

For details, see e.g., $[4,6-9,16,17]$.

The formula (1.3) led the author to consider the computation of the 2-norm joint spectral radius for general matrices. This led to the result for $\rho_{2}(\mathcal{A})$ in Theorem 1 (with $k=1$ ).

Recently, Lau and Ma [19] showed that the critical $L_{p}$ regularity of solutions of refinement equations with 4-coefficients can be computed in terms of the spectral radius of some finite matrix when $p$ is an even integer. Their essential goal was to compute the $2 k$-norm $(k \in \mathbb{N})$ joint spectral radius of two $2 \times 2$ matrices. Although these $2 \times 2$ matrices have special structures, the result in [19] in connection with the case $k=1$ of Theorem 1 prompted the author to study the $2 k$-norm $(k \in \mathbb{N})$ joint spectral radius for general multisets of matrices.

The purpose of this paper is to provide a formula for the p-norm joint spectral radius when $p$ is an even integer. We do not assume any special structures on the matrices and the result is stated in terms of the spectral radius of some finite matrix. Our result is new even in the well-known case $p=2$. We hope that it will be useful to fields other than wavelet analysis.

To state our main result, we need the concept of the Kronecker product (see, e.g., [18]). The Kronecker product of $A \in \mathbb{C}^{m \times m}$ and $B \in \mathbb{C}^{n \times n}$ is the block matrix

$$
A \otimes B:=\left[\begin{array}{cccc}
a_{11} B & a_{12} B & \cdots & a_{1 m} B \\
a_{21} B & a_{22} B & \cdots & a_{2 m} B \\
\vdots & \vdots & \ddots & \vdots \\
a_{m 1} B & a_{m 2} B & \cdots & a_{m m} B
\end{array}\right] \in \mathbb{C}^{m n \times m n}
$$

Set $A^{[1]}=A$ and $A^{[l+1]}:=A \otimes A^{[l]}$ for $l \in \mathbb{N}$. Hence, $A^{[l]} \in \mathbb{C}^{m^{l} \times m^{l}}$. Denote $\bar{A}=\left(\bar{a}_{j l}\right)_{j, l=1}^{m}, A^{T}=\left(a_{l j}\right)_{j, l=1}^{m}$, and $A^{*}=\left(\bar{a}_{l j}\right)_{j, l=1}^{m}$. Note that $(A \otimes B)^{T}=A^{T} \otimes B^{T}$. 
Using these notations, our main result can be stated as follows.

Theorem 1. Let $\mathcal{A}=\left\{A_{1}, \ldots, A_{d}\right\}$ be a multiset of $M \times M$ matrices, $k \in \mathbb{N}$ and let $F \in \mathbb{C}^{M^{2 k} \times M^{2 k}}$ be defined by

$$
F:=\sum_{l=1}^{d}\left(\bar{A}_{l} \otimes A_{l}\right)^{[k]} .
$$

Then

$$
\rho_{2 k}(\mathcal{A})=(\rho(F))^{1 / 2 k} .
$$

Moreover, there is a positive constant $C$ such that for all $n \in \mathbb{N}$,

$$
\left\|\mathcal{A}^{n}\right\|_{2 k} \leq C\left(\rho_{2 k}(\mathcal{A})\right)^{n}
$$

if and only if all the eigenvalues of $F$ with modulus equal to $\rho(F)$ are nondegenerate.

Although Theorem 1 only deals with the $2 k$-norm joint spectral radius, it provides us some information about the uniform joint spectral radius. The following nice equivalence is one example:

$$
\rho_{\infty}(\mathcal{A})=\rho_{2}(\mathcal{A}) \quad \Longleftrightarrow \quad \rho_{4}(\mathcal{A})=\rho_{2}(\mathcal{A})
$$

More connections to uniform joint spectral radius and applications for multiple refinable functions will be presented in Section 4.

The proof of Theorem 1 will be given in Section 3 following some discussion and using lemmas from Section 2.

\section{Kronecker product and string vec-function}

In this section, we introduce the concept of string vec-function and provide its connection to the Kronecker product.

For an $m \times n$ complex matrix $A$, write $A=\left[A_{* 1} \cdots A_{* n}\right]$, where for $j=1, \ldots, n$, $A_{* j} \in \mathbb{C}^{m}$ stands for the $j$ th column vector of $A$. Then the vector

$$
\operatorname{vec} A:=\left[\begin{array}{c}
A_{* 1} \\
A_{* 2} \\
\vdots \\
A_{* n}
\end{array}\right] \in \mathbb{C}^{m n}
$$

is called the vec-function of $A$. It is the vector formed by stacking the columns of $A$ into one long vector.

The following result (see, e.g., [18]) indicates a close relation between the vecfunction and the Kronecker product.

Lemma 1. If $A \in \mathbb{C}^{m \times m}, B \in \mathbb{C}^{n \times n}, X \in \mathbb{C}^{m \times n}$, then

$$
\operatorname{vec}(A X B)=\left(B^{T} \otimes A\right) \operatorname{vec} X .
$$

The relation in Lemma 1 is often employed to deal with products of noncommutative matrices such as solving the linear matrix equation

$$
A X+X B=C
$$


for the unknown matrix $X$. For our purpose, we also have to deal with products of noncommutative matrices. Hence Lemma 1 plays a crucial role in our analysis for $k=1$. To give the reader some clues, we first consider this case: the 2-norm joint spectral radius. Let $e_{j}$ be the $j$ th column of the identity matrix.

If we choose $\|A\|$ as the matrix norm on $\ell_{\infty}$, then

$$
\|A\|_{\infty}^{2}=\max _{1 \leq j \leq M}\left\|e_{j}^{T} A\right\|_{1}^{2} \leq \max _{1 \leq j \leq M} M\left\|e_{j}^{T} A\right\|_{2}^{2}=\max _{1 \leq j \leq M} M\left(e_{j}^{T} A A^{*} e_{j}\right) .
$$

By the definition of vec-function, $e_{j}^{T} A A^{*} e_{j}=\operatorname{vec}\left(A A^{*}\right)(j+(j-1) M)$. Hence

$$
\|A\|_{\infty}^{2} \leq M \sum_{j=1}^{M} \operatorname{vec}\left(A A^{*}\right)(j+(j-1) M) .
$$

Together with Lemma 1 , this implies that for $A=A_{\varepsilon_{1}} A_{\varepsilon_{2}} \cdots A_{\varepsilon_{n}}$,

$$
\begin{aligned}
& \left\|A_{\varepsilon_{1}} A_{\varepsilon_{2}} \cdots A_{\varepsilon_{n}}\right\|_{\infty}^{2} \leq M \sum_{j=1}^{M} \operatorname{vec}\left(A_{\varepsilon_{1}} A_{\varepsilon_{2}} \cdots A_{\varepsilon_{n}} A_{\varepsilon_{n}}^{*} A_{\varepsilon_{n-1}}^{*} \cdots A_{\varepsilon_{1}}^{*}\right)(j+(j-1) M) \\
& \quad=M \sum_{j=1}^{M}\left[\left(\bar{A}_{\varepsilon_{1}} \otimes A_{\varepsilon_{1}}\right) \cdots\left(\bar{A}_{\varepsilon_{n}} \otimes A_{\varepsilon_{n}}\right) \operatorname{vec}\left(I_{M \times M}\right)\right](j+(j-1) M) .
\end{aligned}
$$

Applying this estimate in the definition, we obtain

$$
\left\|\mathcal{A}^{n}\right\|_{2}^{2} \leq M \sum_{j=1}^{M}\left[\left(\sum_{l=1}^{d} \bar{A}_{l} \otimes A_{l}\right)^{n} \operatorname{vec}\left(I_{M \times M}\right)\right](j+(j-1) M) .
$$

Observe that for $k=1$, the matrix $F$ in Theorem 1 equals $\sum_{l=1}^{d} \bar{A}_{l} \otimes A_{l}$. Therefore, for every $n \in \mathbb{N}$,

$$
\left\|\mathcal{A}^{n}\right\|_{2}^{2} \leq M\left\|F^{n} \operatorname{vec}\left(I_{M \times M}\right)\right\|_{1} \leq M^{2}\left\|F^{n}\right\|_{1} .
$$

This provides one direction of the proof for the case $k=1$.

For the other direction, let $Y \in \mathbb{C}^{M \times M}$. Then by Lemma 1

$$
F \operatorname{vec}(Y)=\sum_{\varepsilon=1}^{d}\left(\bar{A}_{\varepsilon} \otimes A_{\varepsilon}\right) \operatorname{vec}(Y)=\sum_{\varepsilon=1}^{d} \operatorname{vec}\left(A_{\varepsilon} Y A_{\varepsilon}^{*}\right)
$$

Hence,

$$
F^{n} \operatorname{vec}(Y)=\sum_{\varepsilon_{1}=1}^{d} \cdots \sum_{\varepsilon_{n}=1}^{d} \operatorname{vec}\left(A_{\varepsilon_{1}} A_{\varepsilon_{2}} \cdots A_{\varepsilon_{n}} Y A_{\varepsilon_{n}}^{*} A_{\varepsilon_{n-1}}^{*} \cdots A_{\varepsilon_{1}}^{*}\right)
$$

Let $j, l \in\{1, \ldots, M\}$. We choose $Y=e_{j} e_{l}^{T} \in \mathbb{C}^{M \times M}$. Then $\operatorname{vec}(Y)=e_{l+(j-1) M} \in$ $\mathbb{C}^{M^{2}}$ and $A Y A^{*}=A e_{j}\left(A e_{l}\right)^{*}$. The above expression for $F^{n}$ tells us that

$$
F^{n} e_{l+(j-1) M}=\sum_{\varepsilon_{1}, \ldots, \varepsilon_{n} \in\{1, \ldots, d\}} \operatorname{vec}\left(\left[A_{\varepsilon_{1}} A_{\varepsilon_{2}} \cdots A_{\varepsilon_{n}} e_{j}\right]\left[A_{\varepsilon_{1}} A_{\varepsilon_{2}} \cdots A_{\varepsilon_{n}} e_{l}\right]^{*}\right) .
$$

Note that for $u, v \in \mathbb{C}^{M}$,

$$
\left\|\operatorname{vec}\left(u v^{*}\right)\right\|_{1}=\|u\|_{1}\|v\|_{1} .
$$


Therefore,

$$
\begin{aligned}
\left\|F^{n} e_{l+(j-1) M}\right\|_{1} & \leq \sum_{\varepsilon_{1}, \ldots, \varepsilon_{n} \in\{1, \ldots, d\}}\left\|A_{\varepsilon_{1}} A_{\varepsilon_{2}} \cdots A_{\varepsilon_{n}} e_{j}\right\|_{1}\left\|A_{\varepsilon_{1}} A_{\varepsilon_{2}} \cdots A_{\varepsilon_{n}} e_{l}\right\|_{1} \\
& \leq \sum_{\varepsilon_{1}, \ldots, \varepsilon_{n} \in\{1, \ldots, d\}} M^{2}\left\|A_{\varepsilon_{1}} A_{\varepsilon_{2}} \cdots A_{\varepsilon_{n}}\right\|_{\infty}^{2}
\end{aligned}
$$

which implies that

$$
\left\|F^{n}\right\|_{1}=\max _{1 \leq j, l \leq M}\left\|F^{n} e_{l+(j-1) M}\right\|_{1} \leq M^{2}\left\|\mathcal{A}^{n}\right\|_{2}^{2} .
$$

Together with (2.1), this provides us with the inequalities

$$
\frac{1}{M} \sqrt{\left\|F^{n}\right\|_{1}} \leq\left\|\mathcal{A}^{n}\right\|_{2} \leq M \sqrt{\left\|F^{n}\right\|_{1}} .
$$

The proof of Theorem 1 with $k=1$ follows then from these inequalities.

To prove Theorem 1 for general $k$, we need to generalize the vec-function and introduce the concept of string vec-function of a string of matrices.

Let $\mathcal{E}:=\left(E_{1}, \ldots, E_{k}\right)$ be a string of $M \times M$ complex matrices. Define the string vec-function $\operatorname{vec}(\mathcal{E})=\operatorname{vec}\left(E_{1}, \ldots, E_{k}\right)$ as a string of vectors $\left(\operatorname{vec}(\mathcal{E})_{1}, \ldots, \operatorname{vec}(\mathcal{E})_{k}\right)$ by

$$
\operatorname{vec}(\mathcal{E})_{1}=\operatorname{vec} E_{1} \in \mathbb{C}^{M^{2}}
$$

and, for $j=2, \ldots, k$,

$$
\operatorname{vec}(\mathcal{E})_{j}=\operatorname{vec}\left(\operatorname{vec}(\mathcal{E})_{j-1}\left(\operatorname{vec} E_{j}\right)^{T}\right) \in \mathbb{C}^{M^{2 j}} .
$$

The following lemma is a generalization of Lemma 1, providing a relation between the string vec-function and the Kronecker product, and plays the same role for general $k$ as Lemma 1 for $k=1$.

Lemma 2. Let $\left(E_{1}, \ldots, E_{k}\right)$ be a string of $M \times M$ complex matrices and $A \in \mathbb{C}^{M \times M}$. Then the string vec-function of the string $\left(A E_{1} A^{*}, \ldots, A E_{k} A^{*}\right)$ satisfies

$$
\operatorname{vec}\left(A E_{1} A^{*}, \ldots, A E_{k} A^{*}\right)_{j}=(\bar{A} \otimes A)^{[j]} \operatorname{vec}\left(E_{1}, \ldots, E_{k}\right)_{j}, \quad j=1, \ldots, k .
$$

Proof. The first component $(j=1)$ is clear by Lemma 1 and the definition of string vec-function:

$$
\begin{aligned}
(\bar{A} \otimes A)^{[1]} \operatorname{vec}\left(E_{1}, \ldots, E_{k}\right)_{1} & =(\bar{A} \otimes A) \operatorname{vec} E_{1}=\operatorname{vec}\left(A E_{1} A^{*}\right) \\
& =\operatorname{vec}\left(A E_{1} A^{*}, \ldots, A E_{k} A^{*}\right)_{1} .
\end{aligned}
$$

Suppose that (2.2) holds for $j=s-1$. Then by the definition of string vec-function, $(\bar{A} \otimes A)^{[s]} \operatorname{vec}\left(E_{1}, \ldots, E_{k}\right)_{s}$

$$
=\left[(\bar{A} \otimes A) \otimes(\bar{A} \otimes A)^{[s-1]}\right] \operatorname{vec}\left(\operatorname{vec}\left(E_{1}, \ldots, E_{k}\right)_{s-1}\left(\operatorname{vec} E_{s}\right)^{T}\right) .
$$

According to Lemma 1 , this equals

$$
\operatorname{vec}\left((\bar{A} \otimes A)^{[s-1]} \operatorname{vec}\left(E_{1}, \ldots, E_{k}\right)_{s-1}\left(\operatorname{vec} E_{s}\right)^{T}(\bar{A} \otimes A)^{T}\right) .
$$

By our induction hypothesis,

$$
(\bar{A} \otimes A)^{[s-1]} \operatorname{vec}\left(E_{1}, \ldots, E_{k}\right)_{s-1}=\operatorname{vec}\left(A E_{1} A^{*}, \ldots, A E_{k} A^{*}\right)_{s-1},
$$

while Lemma 1 implies

$$
\left(\operatorname{vec} E_{s}\right)^{T}(\bar{A} \otimes A)^{T}=\left([\bar{A} \otimes A] \operatorname{vec} E_{s}\right)^{T}=\left(\operatorname{vec}\left(A E_{s} A^{*}\right)\right)^{T} .
$$


Therefore,

$$
(\bar{A} \otimes A)^{[s]} \operatorname{vec}\left(E_{1}, \ldots, E_{k}\right)_{s}=\operatorname{vec}\left(\operatorname{vec}\left(A E_{1} A^{*}, \ldots, A E_{k} A^{*}\right)_{s-1}\left(\operatorname{vec}\left(A E_{s} A^{*}\right)\right)^{T}\right) .
$$

By the definition of string vec-function, it equals exactly

$$
\operatorname{vec}\left(A E_{1} A^{*}, \ldots, A E_{k} A^{*}\right)_{s} .
$$

Hence, (2.2) is valid for $s$. This completes the induction procedure.

Let $k \in \mathbb{N}$ and $\left\{A_{1}, \ldots, A_{d}\right\}$ be a multiset of $M \times M$ complex matrices. For $\varepsilon=1, \ldots, d$, define

$$
F_{\varepsilon}:=\left(\bar{A}_{\varepsilon} \otimes A_{\varepsilon}\right)^{[k]}
$$

Then Lemma 2 implies that

$$
F_{\varepsilon} \operatorname{vec}\left(E_{1}, \ldots, E_{k}\right)_{k}=\operatorname{vec}\left(A_{\varepsilon} E_{1} A_{\varepsilon}^{*}, \ldots, A_{\varepsilon} E_{k} A_{\varepsilon}^{*}\right)_{k} .
$$

Applying this formula $n$ times, we have for $\varepsilon_{1}, \ldots, \varepsilon_{n} \in\{1, \ldots, d\}$,

$$
F_{\varepsilon_{1}} \cdots F_{\varepsilon_{n}} \operatorname{vec}\left(E_{1}, \ldots, E_{k}\right)_{k}=\operatorname{vec}(\mathcal{E})_{k} \text {. }
$$

For convenience, we have denoted $\mathcal{E}$ as the string of matrices

$$
\mathcal{E}:=\operatorname{vec}\left(A_{\varepsilon_{1}} A_{\varepsilon_{2}} \cdots A_{\varepsilon_{n}} E_{1} A_{\varepsilon_{n}}^{*} A_{\varepsilon_{n-1}}^{*} \cdots A_{\varepsilon_{1}}^{*}, \ldots, A_{\varepsilon_{1}} A_{\varepsilon_{2}} \cdots A_{\varepsilon_{n}} E_{k} A_{\varepsilon_{n}}^{*} A_{\varepsilon_{n-1}}^{*} \cdots A_{\varepsilon_{1}}^{*}\right) \text {. }
$$

Note that for $u \in \mathbb{C}^{m}, v \in \mathbb{C}^{r}, 1 \leq j \leq m, 1 \leq l \leq r$,

$$
e_{j+(l-1) m}^{T} \operatorname{vec}\left(u v^{T}\right)=\left(e_{j}^{T} u\right)\left(e_{l}^{T} v\right) .
$$

Together with the definition of string vec-function, the formula (2.4) tells us that for $s=2, \ldots, k, 1 \leq j \leq M^{2(s-1)}, 1 \leq l \leq M^{2}$,

$$
e_{j+(l-1) M^{2(s-1)}}^{T} \operatorname{vec}(\mathcal{E})_{s}=\left[e_{j}^{T} \operatorname{vec}(\mathcal{E})_{s-1}\right]\left[e_{l}^{T} \operatorname{vec}\left(A_{\varepsilon_{1}} A_{\varepsilon_{2}} \cdots A_{\varepsilon_{n}} E_{s} A_{\varepsilon_{n}}^{*} A_{\varepsilon_{n-1}}^{*} \cdots A_{\varepsilon_{1}}^{*}\right)\right] \text {. }
$$

Apply this formula recursively for $s=k, k-1, \ldots, 2$. Then in connection with (2.3), we obtain the following lemma.

Lemma 3. Let $\left(E_{1}, \ldots, E_{k}\right)$ be a string of $M \times M$ complex matrices. Then for $l_{1}, \ldots, l_{2 k} \in\{1, \ldots, M\}$ and $\varepsilon_{1}, \ldots, \varepsilon_{n} \in\{1, \ldots, d\}$, we have

$$
\begin{aligned}
& e_{1}^{T}+\sum_{s=1}^{2 k}\left(l_{s}-1\right) M^{s-1} \\
& \quad F_{\varepsilon_{1}} \cdots F_{\varepsilon_{n}} \operatorname{vec}\left(E_{1}, \ldots, E_{k}\right)_{k} \\
& \quad=\prod_{s=1}^{k}\left\{e_{l_{2 s-1}+\left(l_{2 s}-1\right) M}^{T}\left[\operatorname{vec}\left(A_{\varepsilon_{1}} A_{\varepsilon_{2}} \cdots A_{\varepsilon_{n}} E_{s} A_{\varepsilon_{n}}^{*} A_{\varepsilon_{n-1}}^{*} \cdots A_{\varepsilon_{1}}^{*}\right)\right]\right\} .
\end{aligned}
$$

\section{Proof of Theorem 1}

In this section, we use the preliminary results given in the last section to prove Theorem 1 .

Proof of Theorem 1. We apply Lemma 3 to a special string of matrices. Let $j_{1}, \ldots, j_{2 k}$ $\in\{1, \ldots, M\}$. Define

$$
E_{s}:=e_{j_{2 s-1}} e_{j_{2 s}}^{T} \in \mathbb{C}^{M \times M}, \quad s=1, \ldots, k .
$$

Then

$$
\operatorname{vec} E_{s}=e_{j_{2 s-1}+\left(j_{2 s}-1\right) M}
$$


and, from the definition of string vec-function,

$$
\operatorname{vec}\left(E_{1}, \ldots, E_{k}\right)_{s}=e_{1+\sum_{t=1}^{2 s}\left(j_{t}-1\right) M^{t-1}}
$$

Moreover, by (2.4),

$$
\begin{array}{r}
e_{l_{2 s-1}}^{T}+\left(l_{2 s}-1\right) M \\
=\left[\operatorname{vec}\left(A_{\varepsilon_{1}} A_{\varepsilon_{2}} \cdots A_{\varepsilon_{n}} E_{s} A_{\varepsilon_{n}}^{*} A_{\varepsilon_{n-1}}^{*} \cdots A_{\varepsilon_{1}}^{*}\right)\right] \\
=\left[e_{l_{2 s-1}}^{T} A_{\varepsilon_{1}} A_{\varepsilon_{2}} \cdots A_{\varepsilon_{n}} e_{j_{2 s-1}}\right]\left[e_{l_{2 s}}^{T} \bar{A}_{\varepsilon_{1}} \cdots \bar{A}_{\varepsilon_{n}} e_{j_{2 s}}\right] .
\end{array}
$$

This, in connection with (3.1) and Lemma 3, implies

$$
\begin{aligned}
& e_{1+\sum_{s=1}^{2 k}\left(l_{s}-1\right) M^{s-1}}^{T} F_{\varepsilon_{1}} \cdots F_{\varepsilon_{n}} e_{1+\sum_{s=1}^{2 k}\left(j_{s}-1\right) M^{s-1}} \\
& \quad=\prod_{s=1}^{k}\left\{\left[e_{l_{2 s-1}^{T}}^{T} A_{\varepsilon_{1}} A_{\varepsilon_{2}} \cdots A_{\varepsilon_{n}} e_{j_{2 s-1}}\right]\left[e_{l_{2 s}}^{T} \bar{A}_{\varepsilon_{1}} \cdots \bar{A}_{\varepsilon_{n}} e_{j_{2 s}}\right]\right\} .
\end{aligned}
$$

Now we use this formula to estimate the norms. As in the discussion for the case $k=1$, applying Hölder's inequality, we have in one direction

$$
\begin{aligned}
\left\|A_{\varepsilon_{1}} A_{\varepsilon_{2}} \cdots A_{\varepsilon_{n}}\right\|_{\infty}^{2 k} & =\max _{1 \leq l \leq M}\left\|e_{l}^{T} A_{\varepsilon_{1}} A_{\varepsilon_{2}} \cdots A_{\varepsilon_{n}}\right\|_{l_{1}}^{2 k} \\
& \leq \max _{1 \leq l \leq M} M^{2 k-1} \sum_{j=1}^{M}\left|e_{l}^{T} A_{\varepsilon_{1}} A_{\varepsilon_{2}} \cdots A_{\varepsilon_{n}} e_{j}\right|^{2 k} \\
& \leq \sum_{l=1}^{M} M^{2 k-1} \sum_{j=1}^{M} \prod_{s=1}^{k}\left\{\left[e_{l}^{T} A_{\varepsilon_{1}} A_{\varepsilon_{2}} \cdots A_{\varepsilon_{n}} e_{j}\right]\left[e_{l}^{T} \bar{A}_{\varepsilon_{1}} \cdots \bar{A}_{\varepsilon_{n}} e_{j}\right]\right\} \\
& =M^{2 k-1} \sum_{l=1}^{M} \sum_{j=1}^{M} e_{1+\sum_{s=1}^{2 k}(l-1) M^{s-1}}^{T} F_{\varepsilon_{1}} \cdots F_{\varepsilon_{n}} e_{1+\sum_{s=1}^{2 k}(j-1) M^{s-1}} .
\end{aligned}
$$

Note that $F=F_{1}+\cdots+F_{d}$. Hence

$$
\begin{aligned}
\left\|\mathcal{A}^{n}\right\|_{2 k}^{2 k} & :=\sum_{\varepsilon_{1}, \ldots, \varepsilon_{n} \in\{1, \ldots, d\}}\left\|A_{\varepsilon_{1}} A_{\varepsilon_{2}} \cdots A_{\varepsilon_{n}}\right\|_{\infty}^{2 k} \\
& \leq M^{2 k-1} \sum_{l=1}^{M} \sum_{j=1}^{M} e_{1+\sum_{s=1}^{2 k}(l-1) M^{s-1}}^{T}\left(\sum_{\varepsilon=1}^{d} F_{\varepsilon}\right)^{n} e_{1+\sum_{s=1}^{2 k}(j-1) M^{s-1}} \\
& \leq M^{2 k}\left\|F^{n}\right\|_{1} .
\end{aligned}
$$

For the other direction, letting $j:=1+\sum_{s=1}^{2 k}\left(j_{s}-1\right) M^{s-1} \in\left\{1, \ldots, M^{2 k}\right\}$ where each $j_{s}$ is from $\{1, \ldots, M\}$, we have

$$
\begin{aligned}
\left\|F^{n} e_{j}\right\|_{l_{1}} & \leq \sum_{\varepsilon_{1}, \ldots, \varepsilon_{n} \in\{1, \ldots, d\}}\left\|F_{\varepsilon_{1}} \cdots F_{\varepsilon_{n}} e_{j}\right\|_{l_{1}} \\
& \leq \sum_{\varepsilon_{1}, \ldots, \varepsilon_{n} \in\{1, \ldots, d\}} M^{2 k} \max _{1 \leq l \leq M^{2 k}}\left|e_{l}^{T} F_{\varepsilon_{1}} \cdots F_{\varepsilon_{n}} e_{j}\right| \\
& \leq \sum_{\varepsilon_{1}, \ldots, \varepsilon_{n} \in\{1, \ldots, d\}} M^{2 k}\left\|A_{\varepsilon_{1}} A_{\varepsilon_{2}} \cdots A_{\varepsilon_{n}}\right\|_{\infty}^{2 k} \\
& =M^{2 k}\left\|\mathcal{A}^{n}\right\|_{2 k}^{2 k} .
\end{aligned}
$$


Combining the above estimates for the two directions, we obtain for $n \in \mathbb{N}$,

$$
\frac{1}{M}\left\|F^{n}\right\|_{1}^{1 / 2 k} \leq\left\|\mathcal{A}^{n}\right\|_{2 k} \leq M\left\|F^{n}\right\|_{1}^{1 / 2 k}
$$

This implies the conclusion of Theorem 1, and our proof is complete.

\section{Uniform joint spectral radius}

Our main result, Theorem 1 , tells us that the $p$-norm joint spectral radius can be computed by the spectral radius of a finite matrix $F$ when $p=2 k$ is an even integer. Hence, it can be applied to the sharp estimates of $L_{p}$-smoothness of wavelets with $p$ being an even integer.

By the definition of $\rho_{p}(\mathcal{A})$, we can easily see that for $k \in \mathbb{N}$,

$$
\rho_{\infty}(\mathcal{A}) \leq \rho_{2 k}(\mathcal{A}) \leq d^{1 / 2 k} \rho_{\infty}(\mathcal{A}) .
$$

Hence, we may estimate the uniform joint spectral radius by the spectral radii of finite matrices with the error given by (4.1). However, since the order of the matrix $F$ is $M^{2 k}$, which increases very fast as $k$ tends to infinity, it is difficult to apply this method to compute the uniform joint spectral radius exactly. A more efficient way to get $\rho_{\infty}(\mathcal{A})$ is to apply the relation

$$
\rho_{\infty}(\mathcal{A})=\lim _{n \rightarrow \infty}\left[\max \left\{\rho\left(A_{\varepsilon_{1}} A_{\varepsilon_{2}} \cdots A_{\varepsilon_{n}}\right): A_{\varepsilon_{1}}, \ldots, A_{\varepsilon_{n}} \in \mathcal{A}\right\}\right]^{1 / n}
$$

proved in [1] (see also [3]). However, it is difficult to give an error bound for this approximation process. Let us mention that the finiteness conjecture, i.e.,

$$
\rho_{\infty}(\mathcal{A})=\left[\max \left\{\rho\left(A_{\varepsilon_{1}} A_{\varepsilon_{2}} \cdots A_{\varepsilon_{n}}\right): A_{\varepsilon_{1}}, \ldots, A_{\varepsilon_{n}} \in \mathcal{A}\right\}\right]^{1 / n} \text { for some } n
$$

remains open.

Observe that for $k=1$, the order of $F$ is $M^{2}$, while we know (see, e.g., $[8,17]$ ) that for the multiset $\mathcal{A}$ arising from wavelets, $\rho_{2}(\mathcal{A})$ equals the square root of the spectral radius of a finite matrix whose order is about $2 M$. Thus, employing the special structure of the matrices in $\mathcal{A}$, arising from wavelets, it is possible to reduce the order of the matrix $F$. This needs further investigation and will be discussed elsewhere.

On the other hand, in some cases, Theorem 1 can still be used to obtain some interesting applications for the uniform joint spectral radius. This is our purpose in this section.

The first application is the computation of the uniform joint spectral radius when the matrices have some special forms.

Example 1. Let

$$
A_{1}=\left[\begin{array}{cc}
\lambda_{1} & 0 \\
\lambda_{2} & 0
\end{array}\right] \quad \text { and } \quad A_{2}=\left[\begin{array}{ll}
0 & \mu_{1} \\
0 & \mu_{2}
\end{array}\right]
$$

where $\lambda_{1}, \lambda_{2}, \mu_{1}, \mu_{2}$ are complex numbers. 
Observe that

$$
\begin{aligned}
B_{1}:=\bar{A}_{1} \otimes A_{1}= & {\left[\begin{array}{llll}
\left|\lambda_{1}\right|^{2} & 0 & 0 & 0 \\
\bar{\lambda}_{1} \lambda_{2} & 0 & 0 & 0 \\
\bar{\lambda}_{2} \lambda_{1} & 0 & 0 & 0 \\
\left|\lambda_{2}\right|^{2} & 0 & 0 & 0
\end{array}\right], } \\
B_{2}:=\bar{A}_{2} \otimes A_{2} & =\left[\begin{array}{llll}
0 & 0 & 0 & \left|\mu_{1}\right|^{2} \\
0 & 0 & 0 & \bar{\mu}_{1} \mu_{2} \\
0 & 0 & 0 & \bar{\mu}_{2} \mu_{1} \\
0 & 0 & 0 & \left|\mu_{2}\right|^{2}
\end{array}\right] .
\end{aligned}
$$

Let $k \in \mathbb{N}$ and $l_{1}, \ldots, l_{k}, j_{1}, \ldots, j_{k} \in\{1,2,3,4\}$. Then by the definition of the Kronecker product,

$$
\left(B_{1}^{[k]}\right)_{1+\sum_{t=1}^{k}\left(l_{t}-1\right) 4^{t-1}, 1+\sum_{t=1}^{k}\left(j_{t}-1\right) 4^{t-1}}=\prod_{t=1}^{k}\left(B_{1}\right)_{l_{t}, j_{t}}
$$

and

$$
\left(B_{2}^{[k]}\right)_{1+\sum_{t=1}^{k}\left(l_{t}-1\right) 4^{t-1}, 1+\sum_{t=1}^{k}\left(j_{t}-1\right) 4^{t-1}}=\prod_{t=1}^{k}\left(B_{2}\right)_{l_{t}, j_{t}} .
$$

Hence, $\left(B_{1}^{[k]}\right)_{l, j} \neq 0$ implies $j=1$, while $\left(B_{2}^{[k]}\right)_{l, j} \neq 0$ only if $j=4^{k}$. Therefore,

$$
F:=B_{1}^{[k]}+B_{2}^{[k]}=\left[\begin{array}{ccccc}
\left|\lambda_{1}\right|^{2 k} & 0 & \cdots & 0 & \left|\mu_{1}\right|^{2 k} \\
* & 0 & \cdots & 0 & * \\
\vdots & \vdots & & \vdots & \vdots \\
* & 0 & \cdots & 0 & * \\
\left|\lambda_{2}\right|^{2 k} & 0 & \cdots & 0 & \left|\mu_{2}\right|^{2 k}
\end{array}\right] .
$$

By Theorem 1, we obtain

$$
\rho_{2 k}\left(\left\{A_{1}, A_{2}\right\}\right)=\left\{\left(\left|\lambda_{1}\right|^{2 k}+\left|\mu_{2}\right|^{2 k}+\sqrt{\left(\left|\lambda_{1}\right|^{2 k}-\left|\mu_{2}\right|^{2 k}\right)^{2}+4\left|\mu_{1}\right|^{2 k}\left|\lambda_{2}\right|^{2 k}}\right) / 2\right\}^{1 / 2 k} \cdots
$$

Therefore, by (4.1),

$$
\rho_{\infty}\left(\left\{A_{1}, A_{2}\right\}\right)=\lim _{k \rightarrow \infty} \rho_{2 k}\left(\left\{A_{1}, A_{2}\right\}\right)=\max \left\{\left|\lambda_{1}\right|,\left|\mu_{2}\right|, \sqrt{\left|\mu_{1} \lambda_{2}\right|}\right\} .
$$

A special form of Example 1 with $\lambda_{1}=\mu_{2}=t+1 / 2, \lambda_{2}=\mu_{1}=t$ appeared in [17, Example 5.2].

The second application is to obtain some better estimates than that in (4.1).

As an example, for $1 \leq p<\infty, q>0$, from the definition of $\rho_{p}(\mathcal{A})$, we have

$$
\rho_{\infty}(\mathcal{A}) \geq\left(\frac{\rho_{p+q}(\mathcal{A})}{\rho_{p}(\mathcal{A})}\right)^{p / q} \rho_{p+q}(\mathcal{A})
$$


The estimate (4.2) has two advantages: firstly, we can choose various parameters for $p, q$ to optimize the bound in some region; secondly, the choice of $p=q=2$ provides a better estimate than (4.1) with $k=1$ (often used for estimating uniform smoothness of wavelets), since $\rho_{4}(\mathcal{A}) / \rho_{2}(\mathcal{A}) \geq d^{-1 / 4}$.

Together with Theorem 1, (4.2) can be used to check when the first inequality on (4.1) becomes an equality.

Theorem 2. Let $\mathcal{A}$ be a multiset of $M \times M$ complex matrices. Then $\rho_{\infty}(\mathcal{A})=\rho_{2}(\mathcal{A})$ if and only if

$$
\rho_{4}(\mathcal{A})=\rho_{2}(\mathcal{A})
$$

In this case, for any $2 \leq p \leq \infty$,

$$
\rho_{p}(\mathcal{A})=\rho_{\infty}(\mathcal{A})
$$

Proof. Suppose that $\rho_{\infty}(\mathcal{A})=\rho_{2}(\mathcal{A})$, then by (4.1) and (4.2) with $p=q=2$,

$$
\rho_{\infty}(\mathcal{A}) \leq \rho_{4}(\mathcal{A}) \leq \sqrt{\rho_{2}(\mathcal{A}) \rho_{\infty}(\mathcal{A})}=\rho_{2}(\mathcal{A}) .
$$

Hence,

$$
\rho_{4}(\mathcal{A})=\rho_{2}(\mathcal{A})=\rho_{\infty}(\mathcal{A}) .
$$

Conversely, suppose that (4.3) holds, then by (4.1) and (4.2),

$$
\rho_{\infty}(\mathcal{A}) \leq \rho_{2}(\mathcal{A})=\rho_{4}(\mathcal{A}) \leq \sqrt{\rho_{2}(\mathcal{A}) \rho_{\infty}(\mathcal{A})}
$$

Hence,

$$
\rho_{\infty}(\mathcal{A})=\rho_{2}(\mathcal{A})
$$

The second statement (4.4) follows directly from (4.2). The proof of Theorem 2 is complete.

Thus, we can determine the first equality in (4.1) for $k=1$ from $\rho_{2}(\mathcal{A})$ and $\rho_{4}(\mathcal{A})$, which can be easily computed by Theorem 1 . The case $\rho_{\infty}(\mathcal{A})=\rho_{2}(\mathcal{A})$ appeared several times in our investigation of smoothness of multiple refinable functions and multiple wavelets $[16,17]$.

Finally, we provide one example to show the importance of the joint spectral radius in the investigation of multiple refinable functions.

An $r$-vector $\phi=\left(\phi_{1}, \ldots, \phi_{r}\right)^{T}$ of functions on $\mathbb{R}$ is called a multiple refinable function if it satisfies the following matrix refinement equation:

$$
\phi(x)=\sum_{\alpha \in \mathbb{Z}} a(\alpha) \phi(2 x-\alpha)
$$

where $a:=\{a(\alpha)\}_{\alpha \in \mathbb{Z}}$ is a sequence of $r \times r$ complex matrices called the refinement mask and $a(\alpha)=0$ except for finitely many $\alpha$.

In [17], we characterized the smoothness of multiple refinable functions in terms of $p$-norm joint spectral radius. As an application, we have 
Theorem 3. Let $a$ be a finitely supported sequence of $r \times r$ complex matrices and $\phi=\left(\phi_{1}, \ldots, \phi_{r}\right)^{T}$ be a vector of continuous functions satisfying $(4.5)$. If $\phi \in\left(C^{l}(\mathbb{R})\right)^{r}$ for some $l \in \mathbb{Z}_{+}$and $\phi$ has stable shifts, i.e., for two positive constants $C_{1}$ and $C_{2}$,

$$
C_{1} \sum_{j=1}^{r}\left\|b_{j}\right\|_{\infty} \leq\left\|\sum_{j=1}^{r} \sum_{\alpha \in \mathbb{Z}} b_{j}(\alpha) \phi_{j}(x-\alpha)\right\|_{\infty} \leq C_{2} \sum_{j=1}^{r}\left\|b_{j}\right\|_{\infty}, \quad \forall b_{j} \in l_{\infty}(\mathbb{Z}),
$$

then $\phi^{(l)}:=\left(\phi_{1}^{(l)}, \ldots, \phi_{r}^{(l)}\right)^{T} \in(\operatorname{Lip} \delta)^{r}$ for some $\delta>0$.

Proof. Let $\left(\ell_{0}(\mathbb{Z})\right)^{r}$ be the linear space of all finitely supported sequences of $r \times 1$ vectors. Define two linear operators $A_{\varepsilon}, \varepsilon=0,1$, on $\left(\ell_{0}(\mathbb{Z})\right)^{r}$ by

$$
A_{\varepsilon} v(\alpha)=\sum_{\beta \in \mathbb{Z}} a(\varepsilon+2 \alpha-\beta) v(\beta), \quad \alpha \in \mathbb{Z}, \quad v \in\left(\ell_{0}(\mathbb{Z})\right)^{r} .
$$

Suppose $a$ is supported in $\left[N_{1}, N_{2}\right]$, then $(\ell([m, j]))^{r}$, the subspace of $\left(\ell_{0}(\mathbb{Z})\right)^{r}$ consisting of all sequences finitely supported in $[m, j]$ with $m \leq N_{1}, j \geq N_{2}-1$, is invariant under both $A_{0}$ and $A_{1}$. Consequently, the minimal common invariant subspace of $A_{0}$ and $A_{1}$ generated by a finite subset of $\left(\ell_{0}(\mathbb{Z})\right)^{r}$ is finite dimensional. Let $m \in \mathbb{N}$ and $V$ be the minimal common invariant subspace of $A_{0}$ and $A_{1}$ generated by $e_{j}\left(\nabla^{m} \delta\right)$, $j=1, \ldots, r$, where $\delta$ is the delta sequence and $\nabla^{m} \delta=\sum_{s=0}^{m}\left(\begin{array}{c}m \\ s\end{array}\right)(-1)^{s} \delta(\cdot-s)$. Then, $\left[17\right.$, Theorem 3.3, (3.7)] tells us that $-\log _{2} \rho_{\infty}\left(\left.A_{0}\right|_{V},\left.A_{1}\right|_{V}\right)$ is bounded by a fixed number concerning the smoothness of $\phi$. Hence, we can choose $m \in \mathbb{N}$ such that $m>-\log _{2} \rho_{\infty}\left(\left.A_{0}\right|_{V},\left.A_{1}\right|_{V}\right)$ and $m>l$.

Iterating (4.5) $n$ times, we obtain

$$
\phi(x)=\sum_{\alpha \in \mathbb{Z}} a_{n}(\alpha) \phi\left(2^{n} x-\alpha\right)
$$

where $a_{1}=a$ and

$$
a_{n}(\alpha)=\sum_{\beta \in \mathbb{Z}} a_{n-1}(\beta) a(\alpha-2 \beta), \quad \alpha \in \mathbb{Z}
$$

This expression can be found in [16].

It follows that (see [17])

$$
\nabla_{2^{-n}}^{m} \phi(x):=\sum_{s=0}^{m}\left(\begin{array}{c}
m \\
s
\end{array}\right)(-1)^{s} \phi\left(x-s / 2^{n}\right)=\sum_{\alpha \in \mathbb{Z}} \nabla^{m} a_{n}(\alpha) \phi\left(2^{n} x-\alpha\right) .
$$

By the stability assumption, for $1 \leq s \leq r, n \in \mathbb{N}$,

$$
C_{1} \sum_{j=1}^{r}\left\|e_{s}^{T} \nabla^{m} a_{n} e_{j}\right\|_{\infty} \leq\left\|\nabla_{2^{-n}}^{m} \phi_{s}\right\|_{\infty}
$$

Since $\phi \in\left(C^{l}(\mathbb{R})\right)^{r}$ is compactly supported and $m>l$, we know that for $s=$ $1, \ldots, r$,

$$
\lim _{n \rightarrow \infty}\left\|\nabla_{2^{-n}}^{m} \phi_{s}\right\|_{\infty} /\left(2^{-n}\right)^{l}=0 .
$$

This, in connection with $[17,(3.6)]$, implies that for $j=1, \ldots, r$,

$$
\lim _{n \rightarrow \infty} 2^{n l} \max _{\varepsilon_{1}, \ldots, \varepsilon_{n} \in\{0,1\}}\left\{\left\|A_{\varepsilon_{1}} A_{\varepsilon_{2}} \cdots A_{\varepsilon_{n}}\left(e_{j} \nabla^{m} \delta\right)\right\|_{\infty}\right\}=0 .
$$


By the two inequalities in the proof of [9, Lemma 2.4], we have

$$
\lim _{n \rightarrow \infty} 2^{n l} \max _{\varepsilon_{1}, \ldots, \varepsilon_{n} \in\{0,1\}}\left\|\left.\left.A_{\varepsilon_{1}}\right|_{V_{j}} \cdots A_{\varepsilon_{n}}\right|_{V_{j}}\right\|=0, \quad j=1, \ldots, r
$$

where $V_{j}$ is the minimal common invariant subspace of $A_{0}$ and $A_{1}$ generated by $e_{j}\left(\nabla^{m} \delta\right)$.

But

$$
\rho_{\infty}\left(\left\{\left.A_{0}\right|_{V_{j}},\left.A_{1}\right|_{V_{j}}\right\}\right)=\inf _{n \in \mathbb{N}}\left\{\max _{\varepsilon_{1}, \ldots, \varepsilon_{n} \in\{0,1\}}\left\|\left.A_{\varepsilon_{1}}\right|_{V_{j}} \cdots A_{\varepsilon_{n}} \mid V_{j}\right\|\right\}^{1 / n} .
$$

The statement $\rho_{\infty}\left(\left\{\left.A_{0}\right|_{V_{j}},\left.A_{1}\right|_{V_{j}}\right\}\right) \geq 2^{-l}$ would imply a contradiction to (4.7). Therefore, we must have

$$
\rho_{\infty}\left(\left\{\left.A_{0}\right|_{V_{j}},\left.A_{1}\right|_{V_{j}}\right\}\right)<2^{-l}, \quad j=1, \ldots, r .
$$

It follows that

$$
\rho_{\infty}\left(\left\{\left.A_{0}\right|_{V},\left.A_{1}\right|_{V}\right\}\right)<2^{-l}
$$

By [17, Theorem 3.3],

$$
\phi^{(l)} \in(\operatorname{Lip} \delta)^{r}
$$

for some $\delta>0$.

The proof of Theorem 3 is complete.

Remark. Theorem 3 can also be proved by using a factorization of the refinement mask, see e.g., [21]. However, the new mask arising from this factorization has usually a larger support than the original mask. By the structure of shift-invariant spaces, we can show that Theorem 3 still holds without the stability assumption. Also, similar results can be established for $L_{p}$ solutions of (4.5).

Let us now give our example called multiple refinable Hermite interpolants.

Set $r=2$. A vector $\phi=\left(\phi_{1}, \phi_{2}\right)^{T}$ of compactly supported functions is said to be an Hermite interpolant if $\phi_{1}, \phi_{2} \in C^{1}(\mathbb{R})$,

$$
\phi(\alpha)=\delta_{0 \alpha} e_{1}, \quad \alpha \in \mathbb{Z},
$$

and

$$
\phi^{\prime}(\alpha)=\delta_{0 \alpha} e_{2}, \quad \alpha \in \mathbb{Z} .
$$

The following example provides us with a family of multiple refinable Hermite interpolants. Its refinement mask is simple: it is supported in $[0,2]$, but it has rich contents and can be employed to test the power of general theories on matrix refinement equations. We have studied this example several times to show the applicability of our general theories: accuracy in [15], convergence of subdivision schemes in [16], smoothness analysis in [17], and existence of compactly supported distributional solutions in [26].

Example 2. Let $a$ be the sequence of $2 \times 2$ matrices supported in $[0,2]$ given by

$$
a(0)=\left[\begin{array}{cc}
1 / 2 & s / 2 \\
t & 1 / 4+2 s t
\end{array}\right], \quad a(1)=\left[\begin{array}{cc}
1 & 0 \\
0 & 1 / 2
\end{array}\right], \quad \text { and } a(2)=\left[\begin{array}{cc}
1 / 2 & -s / 2 \\
-t & 1 / 4+2 s t
\end{array}\right]
$$

where $s, t$ are real parameters. Then (4.5) has a solution $\phi=\left(\phi_{1}, \phi_{2}\right)^{T}$ such that $\phi(x+1)$ is an Hermite interpolant if and only if $t=-1 / 8,0<s<4$. 
Proof. Let us first prove the following statement:

If $-1 / 2<s t<0, \phi \in\left(C^{1}(\mathbb{R})\right)^{2}$ is supported in $[0,2]$ with $\phi(1)=e_{1}$ and satisfies (4.5) with the mask $a$ given in this example, then

$$
\phi_{2}^{\prime}(1)=-8 t \text {. }
$$

To show this statement, we observe that by $(4.5), \phi(3 / 2)=a(2) \phi(1)=(1 / 2,-t)^{T}$, and for $n \in \mathbb{N}$,

$$
\phi\left(1 / 2^{n}\right)=a(0) \phi\left(1 / 2^{n-1}\right)=\cdots=a(0)^{n} \phi(1)=a(0)^{n} e_{1},
$$

while

$$
\phi\left(1+1 / 2^{n}\right)=a(1) \phi\left(1+1 / 2^{n-1}\right)+a(2) \phi\left(1 / 2^{n-1}\right) .
$$

Hence,

$$
\begin{aligned}
2^{n} \phi_{2}\left(1+1 / 2^{n}\right) & =2^{n-1} \phi_{2}\left(1+1 / 2^{n-1}\right)+2^{n}[-t, 1 / 4+2 s t] a(0)^{n-1} e_{1} \\
& =\cdots=2 \phi_{2}(3 / 2)+\sum_{j=2}^{n} 2^{j}[-t, 1 / 4+2 s t] a(0)^{j-1} e_{1} .
\end{aligned}
$$

If $s t \neq-1 / 8$, the matrix $a(0)$ has two eigenvalues $1 / 4,1 / 2+2 s t$ corresponding to eigenvectors $\xi=(-2 s, 1)^{T}, \eta=(1,4 t)^{T}$, respectively. Then $e_{1}=(-4 t \xi+\eta) /(1+8 s t)$. Thus, in this case,

$$
\begin{aligned}
\phi_{2}^{\prime}(1)= & \lim _{n \rightarrow \infty} \frac{\phi_{2}\left(1+1 / 2^{n}\right)-\phi_{2}(1)}{1 / 2^{n}} \\
= & 2 \phi_{2}(3 / 2)+\sum_{j=2}^{\infty} 2^{j}[-t, 1 / 4+2 s t] \\
& \quad \times\left(-\frac{4 t}{1+8 s t}\left(\frac{1}{4}\right)^{j-1} \xi+\frac{1}{1+8 s t}(1 / 2+2 s t)^{j-1} \eta\right) \\
= & -8 t .
\end{aligned}
$$

If $s t=-1 / 8$, the matrix $a(0)$ has one degenerate eigenvalue $1 / 4$ corresponding to eigenvector $\xi=(-2 s, 1)^{T}$. Also,

$$
a(0) e_{1}=e_{1} / 4-\xi /(8 s) .
$$

Hence,

$$
a(0)^{n} e_{1}=a(0)^{n-1} e_{1} / 4-\left(\frac{1}{4}\right)^{n-1} \xi /(8 s)=\cdots=\left(\frac{1}{4}\right)^{n} e_{1}-n\left(\frac{1}{4}\right)^{n-1} \xi /(8 s) .
$$

Therefore, in this case,

$$
\begin{aligned}
\phi_{2}^{\prime}(1)= & \lim _{n \rightarrow \infty} \frac{\phi_{2}\left(1+1 / 2^{n}\right)-\phi_{2}(1)}{1 / 2^{n}} \\
= & 2 \phi_{2}(3 / 2)+\sum_{j=2}^{\infty} 2^{j}[-t, 1 / 4+2 s t] \\
& \quad \times\left(\left(\frac{1}{4}\right)^{j-1} e_{1}-(j-1)\left(\frac{1}{4}\right)^{j-2} \xi /(8 s)\right) \\
= & -8 t .
\end{aligned}
$$


Combining the above two cases, we conclude that (4.10) holds, and our statement has been proved.

We are in a position to verify the equivalence. Suppose that $\phi$ satisfies (4.5) and $\phi(x+1)$ is an Hermite interpolant, then the shifts of $\phi_{1}$ and $\phi_{2}$ are linearly independent, that is,

$$
\sum_{\alpha \in \mathbb{Z}} b(\alpha) \phi_{1}(x-\alpha)+\sum_{\alpha \in \mathbb{Z}} c(\alpha) \phi_{2}(x-\alpha)=0 \quad \Longrightarrow \quad b(\alpha)=c(\alpha)=0 \quad \forall \alpha \in \mathbb{Z}
$$

This can easily be seen from the Hermite interpolating condition by taking values and derivatives at integers. Hence, the shifts of $\phi_{1}$ and $\phi_{2}$ are stable [14]. This tells us by a result of Dahmen and Micchelli [2] that $|1+4 s t|<2$, i.e., $-3 / 4<s t<1 / 4$. Therefore, we can apply the smoothness analysis from [17, Example 4.2] and conclude from supp $\phi \subset[0,2], \phi \in\left(C^{1}(\mathbb{R})\right)^{2}$ that $|1 / 2+2 s t| \leq 1 / 2$. Moreover, Theorem 3 tells us that $|1 / 2+2 s t|<1 / 2$. Consequently,

$$
-1 / 2<s t<0 \text {. }
$$

Together with the interpolating condition and our statement (4.10), this implies that $t=-1 / 8$ and $0<s<4$, which completes the proof for the necessity part.

Conversely, suppose that $t=-1 / 8,0<s<4$. Then [17, Example 4.2] shows that there is a solution $\phi \in\left(C^{1}(\mathbb{R})\right)^{2}$ of $(4.5)$ such that $\operatorname{supp} \phi \subset[0,2]$ and $\phi(1)=e_{1}$. It follows from our statement (4.10) that $\phi_{2}^{\prime}(1)=1$.

Moreover, we take the derivatives at $x=1$ on both sides of (4.5) and obtain

$$
\phi^{\prime}(1)=a(1) 2 \phi^{\prime}(1)=\left[\begin{array}{ll}
2 & 0 \\
0 & 1
\end{array}\right] \phi^{\prime}(1) .
$$

Hence, $\phi_{1}^{\prime}(1)=0$.

We have shown that $\phi(x+1)$ is an Hermite interpolant, and the proof is complete.

The special case $s=3 / 2, t=-1 / 8, \lambda=-1 / 8$, and $\mu=1 / 2$ was discussed by Heil, Strang, and Strela [11]. In this case, $\phi$ can be obtained explicitly as

$$
\phi_{1}(x)= \begin{cases}x^{2}(-2 x+3) & \text { for } 0 \leq x \leq 1 \\ (2-x)^{2}(2 x-1) & \text { for } 1<x \leq 2 \\ 0 & \text { for } x \in \mathbb{R} \backslash[0,2]\end{cases}
$$

and

$$
\phi_{2}(x)= \begin{cases}x^{2}(x-1) & \text { for } 0 \leq x \leq 1 \\ (2-x)^{2}(x-1) & \text { for } 1<x \leq 2 \\ 0 & \text { for } x \in \mathbb{R} \backslash[0,2]\end{cases}
$$

It is well known that $\phi(x+1)$ is an Hermite interpolant.

When $|1 / 2+2 s t|<2^{-5 / 2}$, if we take $m=2, A_{0}, A_{1}$, and $V$ as in the proof of Theorem 3 , then the joint spectral radius of $\mathcal{A}:=\left\{\left.A_{0}\right|_{V},\left.A_{1}\right|_{V}\right\}$ satisfies $\rho_{\infty}(\mathcal{A})=$ $\rho_{2}(\mathcal{A})=1 / 4$ corresponding to the case in Theorem 2 . On the other hand, if $2^{-2-1 / p}<$ $|1 / 2+2 s t|<2^{-5 / 2}$ for some $1 \leq p<2$, then $\rho_{p}(\mathcal{A})=2^{1 / p}|1 / 2+2 s t|>\rho_{\infty}(\mathcal{A})$. This shows that (4.4) cannot be extended to the range $1 \leq p<2$. 
Acknowledgements. Research supported in part by Research Grants Council of Hong Kong under Grant No. 9040281 and by City University of Hong Kong under Grants Nos. 7000741 and 9030562.

\section{References}

1. M. A. Berger and Y. Wang, Bounded semigroups of matrices, Linear Algebra Appl. 166 (1992), 21-27.

2. W. Dahmen and C. A. Micchelli, Biorthogonal expansions, Constr. Approx. 13 (1997), 293-328.

3. I. Daubechies and J. C. Lagarias, Two-scale difference equations: II. Local regularity, infinite products of matrices and fractals, SIAM J. Math. Anal. 23 (1992), 1031-1079.

4. G. Deslauriers and S. Dubuc, Symmetric iterative interpolation process, Constr. Approx. 5 (1989), 49-68.

5. N. Dyn, J. A. Gregory, and D. Levin, Analysis of uniform binary subdivision schemes for curve design, Constr. Approx. 7 (1991), 127-147.

6. T. Eirola, Sobolev characterization of solutions of dilation equations, SIAM J. Math. Anal. 23 (1992), 1015-1030.

7. T. N. T. Goodman, R. Q. Jia, and C. A. Micchelli, On the spectral radius of a bi-infinite periodic and slanted matrix, preprint.

8. T. N. T. Goodman, C. A. Micchelli, and J. D. Ward, Spectral radius formulas for subdivision operators, In: Recent Advances in Wavelet Analysis, (L. L. Schumaker and G. Webb, eds.), Academic Press, 1994, pp. 335-360.

9. B. Han and R. Q. Jia, Multivariate refinement equations and subdivision schemes, SIAM J. Math. Anal., to appear.

10. C. Heil and D. Colella, Dilation equations and the smoothness of compactly supported wavelets, In: Wavelets: Mathematics, and Applications, (J. Benedetto and M. Frazier, eds.), CRC Press, 1993, pp. 163-201.

11. C. Heil, G. Strang, and V. Strela, Approximation by translates of refinable functions, Numer. Math. 73 (1996), 75-94.

12. R. Q. Jia, Subdivision schemes in $L_{p}$ spaces, Advances in Comp. Math. 3 (1995), 309-341.

13. - Characterization of smoothness of multivariate refinable functions in Sobolev spaces, Trans. Amer. Math. Soc., to appear.

14. R. Q. Jia and C. A. Micchelli, On linear independence of integer translates of a finite number of functions, Proc. Edinburgh Math. Soc. 36 (1992), 69-85.

15. R. Q. Jia, S. Riemenschneider, and D. X. Zhou, Approximation by multiple refinable functions, Canadian J. Math., to appear.

16. _ - Vector subdivision schemes and multiple wavelets, Math. Comp., to appear.

17. _ Smoothness of multiple refinable functions and multiple wavelets, preprint.

18. P. Lancaster and M. Tismenetsky, The Theory of Matrices, Academic Press, 1985.

19. K. S. Lau and M. F. Ma, Regularity of $L^{p}$-scaling functions, Asian J. Math. 1 (1997), 272-292.

20. K. S. Lau and J. Wang, Characterization of $L^{p}$-solutions for the two-scale dilation equations, SIAM J. Math. Anal. 26 (1995), 1018-1046.

21. C. A. Micchelli and T. Sauer, Regularity of multiwavelets, Advances in Comp. Math. 7 (1997), 455-545.

22. G.-C. Rota and G. Strang, A note on the joint spectral radius, Indag. Math. 22 (1960), 379-381.

23. J. N. Tsitsiklis and V. D. Blondel, The Lyapunov exponent and joint spectral radius of pairs of matrices are hard - when not impossible - to compute and to approximate, Math. Control Signals Systems 10 (1997), 31-40.

24. L. F. Villemoes, Wavelet analysis of refinement equations, SIAM J. Math. Anal. 25 (1994), 1433-1460.

25. Y. Wang, Two-scale dilation equations and mean spectral radius, Random Comput. Dynam. 4 (1996), 49-72.

26. D. X. Zhou, Existence of multiple refinable distributions, Michigan J. Math. 44 (1997), 317-329.

Department of Mathematics, City University of Hong Kong, Kowloon Tong, Hong Kong, CHINA

E-mail: mazhou@math.cityu.edu.hk 